\title{
Structural Properties and Surface Plasmon Polariton Propagation of Gold Nanoplates
}

Todd A. Major, Mary Sajini Devadas, Shun Shang Lo, and Gregory V. Hartland

Department of Chemistry and Biochemistry, University of Notre Dame, Notre Dame, Indiana 46556,USA

The optical properties of metal nanoplates offer potential applications, such as in use as sub-wavelength confinement waveguides for lasers with sub-diffraction optical modes [1-2]. One main feature that allows metal nanostructures to be used for those functions is the surface plasmon polariton (SPP), an electromagnetic wave that can be excited at the edge of a nanoparticle by a focused laser beam [3], or through use of a prism [4]. SPPs travel across the surface of the nanoparticle and are re-emitted at the opposite end from the excitation. The propagation length of SPPs can depend on the dielectric properties of the metal, as well as the surface roughness of the nanostructure and the environment [5-6].

In this report, the structure of gold nanoplates synthesized by a polyol procedure [7-9] were analyzed through high resolution Transmission Electron Microscopy (TEM), Scanning Electron Microscopy (SEM), and X-Ray Electron Diffraction (XRD). The gold nanoplates were found to be either hexagonal or triangularly shaped and single crystalline with $\{111\}$ surfaces. The average thickness and length of the nanoplates was found to be $107 \pm 30 \mathrm{~nm}$ and $5 \pm 2 \mu \mathrm{m}$, respectively. The propagation of the SPPs from the synthesized gold nanoplates was studied by focusing a HeNe laser at the edge of the plates and changing the polarization of the beam with a half-wave plate. The direction of the SPPs was found to match well with the polarization of the laser beam.

The author's acknowledgements are listed in reference 10.

References:

[1] R. F. Oulton et al, Nature Photonics 2 (2008), p. 496-500.

[2] R. F. Oulton et al, Nature 461 (2009), p. 629-632.

[3] A. W. Sanders et al, Nano Lett. 6 (2006), p. 1822-1826.

[4] R. M. Dickson, L. A. Lyon, J. Phys. Chem. B 104 (2000), p. 6095-6098.

[5] H. Ditlbacher et al, Phys. Rev. Lett. 95 (2005), p. 257403.

[6] A. Paul et al, ACS Nano 6 (2012), p. 8105-8113.

[7] H. C. Chu, C. H. Kuo, M. H. Huang, Inorg. Chem. 45 (2005), p. 808-813.

[8] Y. Chen et al, J. Chem. Commun. 0, (2005), p. 4181-4183.

[9] H. Liu, Q. Yang, CrystEngComm 13 (2011), p. 2281-2288.

[10] The authors acknowledge funding from the National Science Foundation through Awards CHE1110560 and CHE-0946447 and by the University of Notre Dame Strategic Initiative. The authors would like to thank Dr. Allen Oliver for acquiring and analyzing the XRD data. We also acknowledge the Notre Dame Integrated Imaging Facility for electron microscopy support. 


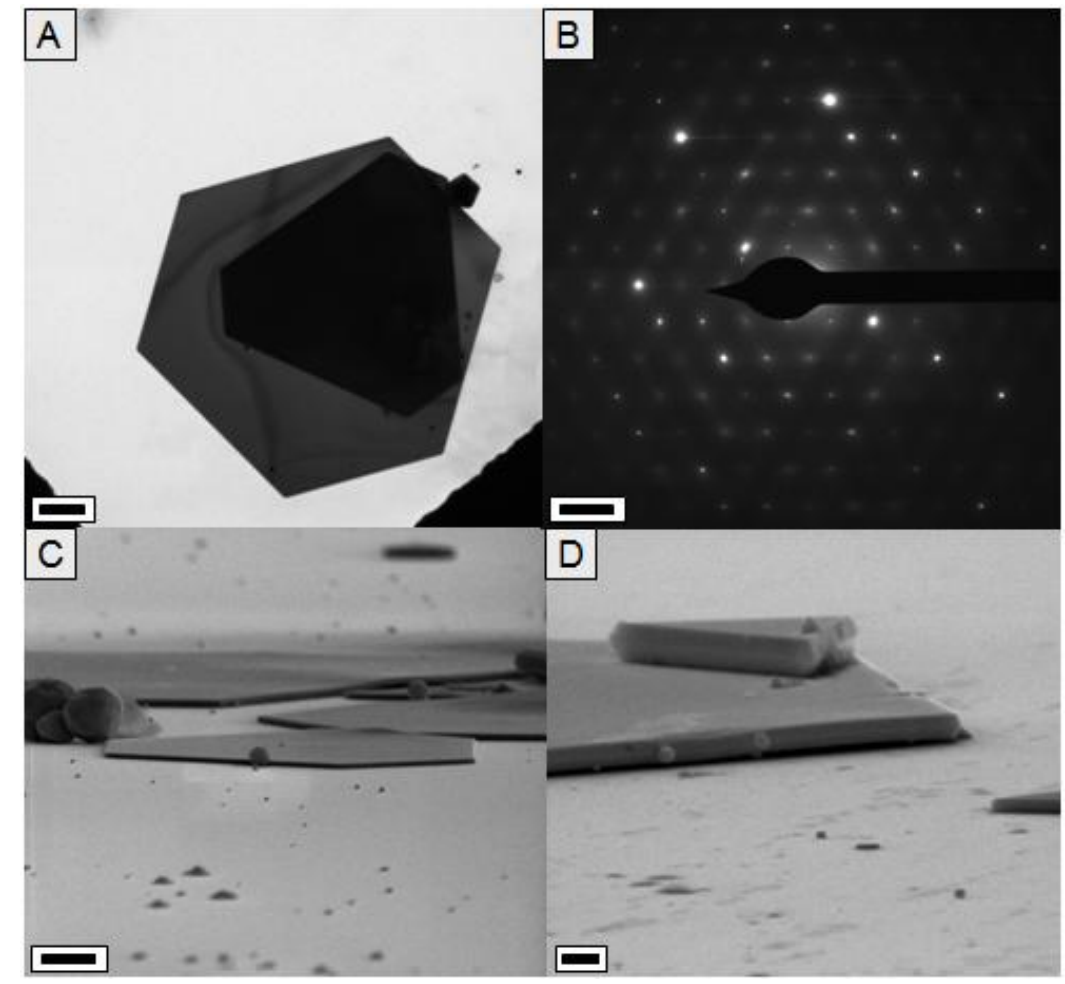

Figure 1. (A): High resolution TEM image of several stacked gold nanoplates. The scale bar corresponds to $1 \mu \mathrm{m}$. (B): Diffraction pattern of a single gold nanoplate. The diffraction pattern clearly represents a $\{111\}$ crystal pattern. The scale bar represents $5 \mathrm{~nm}^{-1}$. (C): Tilted SEM image of an approximately $100 \mathrm{~nm}$ thick gold nanoplate. The scale bar represents $1 \mu \mathrm{m}$. (D) Higher magnification tilted image of several stacked nanoplates. The twinned nature of the nanoplates can be clearly seen from the upper nanoplate. The scale bar represents $200 \mathrm{~nm}$.
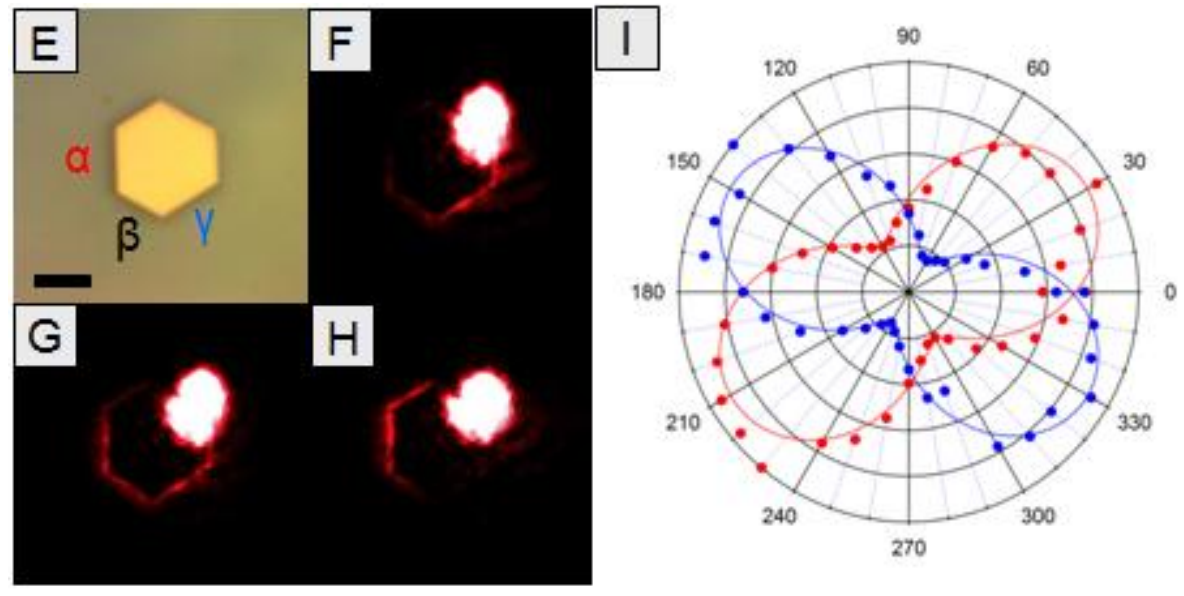

Figure 2. (E) White light scattering light image of a gold nanoplate. The scale bar corresponds to $2 \mu \mathrm{m}$. (B), (C), and (D): Nanoplate in panel A illuminated with a HeNe laser on the top right edge with polarizations of $150^{\circ}, 180^{\circ}$, and $210^{\circ}$, respectively. (E): Polar plot of brightness of the SPP mode as a function of polarization angle. The colors correspond to the sides of the nanoplate labeled as $\alpha$ (red) and $\gamma$ (blue). The red and blue lines represent a sine wave fit to the data. 